\title{
Behavioral Overweight Traits Reflected in DNA Genotype
}

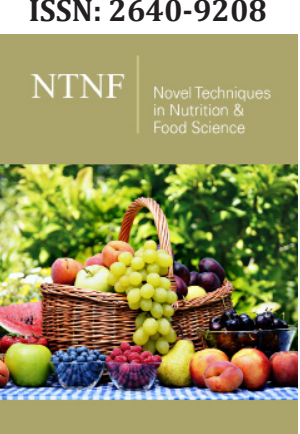

*Corresponding author: Kathy James, Professor of Nursing, Hahn School of Nursing and Health Sciences, USA

Submission: 侮 May 28, 2020

Published: 䟱June 18, 2020

Volume 5 - Issue 1

How to cite this article: Chaunda Celentano, Kathy James, Panagiotis Matsangas, Donna L Agan. Behavioral Overweight Traits Reflected in DNA Genotype. Nov Tech Nutri Food Sci. 5(1). NTNF. 000601. 2020.

DOI: 10.31031/NTNF.2020.05.000601

Copyright@ Kathy James. This article is distributed under the terms of the Creative Commons Attribution 4.0 International License, which permits unrestricted use and redistribution provided that the original author and source are credited.

\author{
Chaunda Celentano ${ }^{1}$, Kathy James ${ }^{2}$, Panagiotis Matsangas ${ }^{3}$ and Donna L Agan ${ }^{4 *}$ \\ ${ }^{1}$ University of San Diego doctoral graduate, San Diego, USA \\ ${ }^{2}$ Professor of Nursing, University of San Diego, Hahn School of Nursing and Health Sciences, \\ USA
}

${ }^{3}$ Lecturer, Operations Research Department, Naval Postgraduate School, Monterey, USA

${ }^{4}$ Lecturer/Consultant, University of San Diego, Hahn School of Nursing and Health Sciences, USA

\begin{abstract}
The use of genetic testing to assist patients with weight loss is relatively new but well received by patients. Genetic testing offers an increased understanding of obesity risk, behaviors that can contribute to weight gain, and more. Research suggests that patients are receptive to learning about how genetics influences their weight. Atypical eating behavior traits (e.g., eating disinhibition, food desire, hunger, satiety, snacking, sweet taste) have been reported in patients with weight problems.

This evidence-based practice project identified the frequency of 6 atypical eating behavior genes in a population of overweight and obese adults. A quantitative retrospective design analyzed behavior traits to identify the frequency of genotypes (e.g., FTO obesity gene) that influenced eating-behavior traits of overweight and obese patients attending a medical weight loss clinic in southern California $(\mathrm{N}=75)$. All study procedures were approved by the appropriate institutional review board and administrators prior to initiating the project. Data were extracted from patients who completed a saliva sample for genomic testing between 2017 and 2018. Analysis revealed that 56(75.7\%) patients screened positive for eating disinhibition and $37(50 \%)$ for food desire, whereas $29(39.2 \%)$ were identified with the FTO gene. Also, $20(27.0 \%)$ patients were positive for sweet taste, $13(17.6 \%)$ satiety, $13(17.6 \%)$ snacking, and $7(9.46 \%)$ hunger. On average, patients screened positive for 2(median) eating behavior traits. Four (5.41\%) patients screened positive for 5 eating behavior traits. Overall, overweight and obese patients had a disproportionally high incidence of eating disinhibition, food desire, and the FTO obese gene; behaviors that could contribute to difficulty with weight loss.
\end{abstract}

This study may provide guidance for advanced practice nurses in how to manage the traits with behavior modification techniques. Specific strategies can be discussed with patients that focus on their atypical behaviors with regular follow up appointments by the clinician. Genetic testing can provide important patient education to improve outcomes related to weight management and health outcomes.

\section{Introduction}

Obesity continues to be a leading problem in the United States. In the latest NHANES Data Report 'Prevalence of Obesity and Severe Obesity Among Adults: United States, 20172018,' the age-adjusted prevalence of obesity among U.S. adults was $42.4 \%$. The prevalence was $40.0 \%$ among younger adults, aged $20-39$ years, $44.8 \%$ among middle-aged adults, aged 40-59 years, and $42.8 \%$ among older adults aged 60 years and older [1]. Causal influences of obesity included lifestyle components (e.g., overeating, poor food choices, lack of exercise) [2]. Extant dietary strategies for weight loss have been designed to reduce calories and increase physical activity. Weight management research and clinical practice suggest differing responses to dietary interventions. Recently, genetic testing has been utilized to analyze associations between genes and obesity risk. Specific genes have been identified that influence eating behaviors as well as the best diet(s) for health and weight loss [3-6]. Eating behavior traits that influence dietary choices have been identified with genetic testing and include snacking, hunger, satiety, eating disinhibition, food desire, and sweet tooth. Genetic information also informs a patient's probable regain of weight loss, adiponectin levels, and metabolism [4,7]. Indeed, genetic evaluation based on metabolic genotypes can clarify which 
dietary and activity recommendations align with long term weight management $[2,3,8]$. Notably, the Doppler and colleagues $[6,7]$ study indicated a 2.5 times greater weight loss with patients receiving genetically tailored diets. Consequently, genetic information may enhance patient success.

The contribution of genetic information has been estimated between $40 \%$ and $70 \%$ of one's predisposition and can be compounded by poor diet or sedentary lifestyle. One's inherited DNA may be as influential on weight as one's environment $[5,6]$. For example, the FTO gene has been associated with fat mass and obesity [5] and evidence suggests that addressing atypical eating behaviors may improve long term weight management, less self-blaming, and greater motivation [9-11]. Arguello et al. [11] examined the existence of atypical eating habits by genotype. This quantitative retrospective study of over 600 patients in weight clinics revealed a high frequency of atypical behaviors around traits (i.e., eating disinhibition, food desire, hunger, satiety, snacking, sweet taste) and an obesity gene. Atypical genotypes in patients with obesity reported eating disinhibition frequency (49\%), food desire (43\%), hunger (3\%), satiety (19\%), and snacking (17\%). As nurse practitioners provide primary care and treatment to patients who are overweight and obese, offering genetic testing as an additional assessment could increase the understanding of underlying contributors. Based on promising results, genetic researchers suggest more robust studies in nutritional genomics given the potential to change dietary disease prevention and therapy to improve public health $[4,7,10,12,13]$.

\section{Framework/EBP model}

The Clinical Scholar Model prepares clinical providers to utilize current research in practice and mentor staff in application [14]. Using genetics in practice is relatively new and can be role modeled by the advanced practice registered nurse (APRN). The process begins with a spirit of inquiry on how diet and genetics can be used to help patients with obesity.

\section{Specific aim}

The purpose of the evidence-based practice project was to identify atypical DNA genotypes of eating behaviors in a selected database of overweight patients who completed genetic testing in a medical weight loss clinic in southern California.

\section{Methods}

\section{Design and participants}

Permission was obtained to use genetic data from a prior study at the clinic and IRB approval was obtained from University of San Diego. Genetic data were reviewed from 75 genetic reports of adult patients who had completed the saliva sample DNA analysis by Pathway Genomics of San Diego, CA between 2017 and 2018. In February 2020, data of the selected genetic behaviors were computed from the deidentified data set of patients who met the criteria for overweight or obese. All patients consented prior to participate in the study. The Pathway Fit DNA test identified over 75 markers related to exercise, metabolic health, nutrition, and food reactions. For this project, eating behavior traits were reviewed and focused on six atypical eating behaviors and the FTO gene obesity allele. The various known atypical eating behavior traits included eating disinhibition, the likelihood to eat more than normal in response to a stimulus; food desire, the amount of effort made to obtain favorite foods; sweet taste, the internal desire for sweets; satiety, the feeling of being satisfied after eating; snacking, the likelihood to show increased snacking behavior; and hunger, the likelihood to report susceptibility to hunger. The coding used for reporting the behavior trait was characterized as typical or atypical behaviors associated with weight gain.

\section{Statistics}

Descriptive statistics were used to analyze the frequency of the six atypical behaviors, obesity gene, age, and sex. The percentages of patients with each atypical genotype was calculated.

\section{Results}

As the database included all overweight and obese individuals, all patients met the criteria. All patients were female between the ages of 22 and 71 years ( $M=47$ years) and high body mass index (BMI $33.2 \pm 5.70 \mathrm{~kg} / \mathrm{m} 2$, range 25.0 to 48.9 ). Behaviors were analyzed for frequency of occurrence: eating disinhibition, food desire, hunger, satiety, snacking, and sweet taste. All patients were assessed for the existence of the obesity gene allele. Four (5.41\%) patients screened positive for five eating behavior traits-the maximum observed in the sample. Specifically, overweight and obese patients had a disproportionally high incidence of eating disinhibition, food desire and the FTO obesity gene (Figure 1).

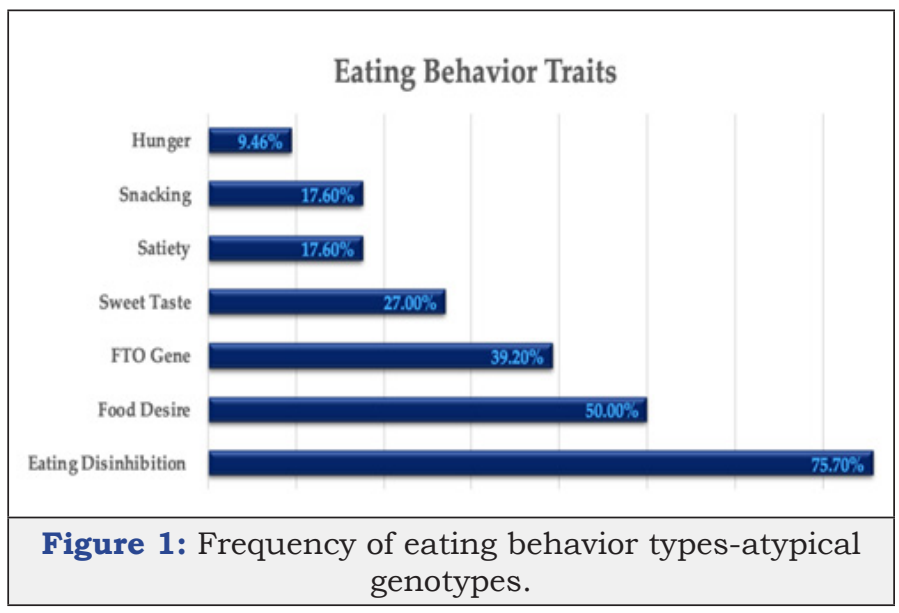

\section{Discussion}

According to the literature, typical genotypes are associated with normal eating behavior traits and atypical genotypes are associated with weight-promoting eating behavior traits [6,7]. The results of this evidence-based practice project are comparable with the prior study by Arguello et al. that looked at many of the same atypical behaviors. This project supports research suggesting that atypical behaviors are common in overweight and obese populations [5]. Although these behaviors can contribute to weight issues, guidance can be provided by the APRN on how to manage these traits with behavior modification techniques. Written guidance will become 
a part of the report that each patient receives in their packet and reviewed by the practitioner [4].

\section{Cost benefit analysis}

The estimated medical cost due to obesity was $\$ 147$ billion in 2019. Medical costs for people with obesity are $\$ 1,429$ higher per year than people with normal weight [1]. The typical cost of a DNA test for the public is from $\$ 200$ to $\$ 300$ [4].

\section{Implications}

Atypical behaviors and the obesity gene can contribute to a patient's difficulty in losing weight. APRNs can provide education to assist patients in understanding known genotype atypical behaviors and utilize specific strategies provided by the DNA genetic report. Thus, genetic testing may provide important patient knowledge to improve outcomes related to weight management and health; however, information does not necessarily equal behavior change. Research suggests new attention to personalized medicine where the universal approach to medical care will evolve into more customized strategies.

With improvements in the cost and efficiency of genome-wide scans and other innovative technologies, health care professionals will be able to utilize these tools to provide patients with individualized information about their risks for certain diseases [15]. New information may enable APRN's to tailor prevention programs for each person's unique genetic structure. The use of genetic testing to assist patients with weight loss is new but has been well received by patients. Genetic testing offers an increased understanding of obesity risk, behaviors that can contribute to weight, and more.

\section{References}

1. USA Department of Health and Human Services, Centers for Disease Control and Prevention, National Center for Health Statistics (2020) Prevalence of obesity and Severe Obesity among adults and youth: United States, 2017-2018. NCHS Data Brief.
2. Centers for Disease Control and Prevention (2017) Adult obesity causes and consequences. Overweight \& Obesity.

3. Eny KM, Wolever TM, Bisson B, Sohemy A (2008) Genetic variant in the glucose transporter type 2 is associated with higher intakes of sugars in two distinct populations. Physiol Genomics 33(3): 355-360.

4. Nova M (2019) Pathway fit scientific validation on dietary information and fit test. Pathway Genomics, San Diego, California, USA.

5. Arkadianos I, Valdes A, Marinos E, Florou A, Gill R, et al. (2007) Improved weight management using genetic information to personalize a caloriecontrolled diet. Nutr J 6(29): 1-8.

6. Morales C, Marsaux CF, Livingstone KM, Carretero SN, Cristobal RS, et al. (2017) Can genetic-based advice help you lose weight? Findings from the Food 4 Me European randomized controlled trial. Am J Clin Nutr 105(5): 1204-1213.

7. Nelson D, Prabakar P, Kondragunta V, Kornman KS, Gardner CD (2010) Genetic phenotypes predict weight loss success: The right diet does matter. Paper presented at: Joint Conference of the 50th Cardiovascular Disease Epidemiology and Prevention and Nutrition, Physical Activity, and Metabolism; San Francisco, California, USA.

8. Ordovas JM (2008) Genotype-phenotype associations: modulation by diet and obesity. Obesity (Silver Spring) 16(3):40-46.

9. Grundy SM (1998) Multifactorial causation of obesity: implications for prevention. Am J Clin Nutr 67(3) (suppl): 563s-572s.

10. Elfhag K, Rössner S (2005) Who succeeds in maintaining weight loss? A conceptual review of factors associated with weight loss maintenance and weight regain. Obes Rev 6(1): 67-85.

11. Arguello LE, Mauldin K, Goyal D (2018) Atypical eating disinhibition genotype. JNP 14(6): 491-496.

12. Meisel SF, Beeken RJ, Jaarsveld CHM, Wardle J (2015) Genetic susceptibility testing and readiness to control weight: Results from a randomized controlled trial. Obesity 23(2): 305-312.

13. Meisel SF, Walker C, Wardle J (2012) Psychological responses to genetic testing for weight gain: A vignette study. Obesity 20(3): 540-546.

14. Remonia E (2016) Evidence-based teaching tactics for frontline staff using the clinical nurse scholar model. JNP 12(1): e1-e5.

15. National Institutes of Health, National Human Genome Research Institute, US Department of Health and Human Services. Genome wide associations fact sheet. 